\title{
INTRODUCING AND SUSTAINING TRADITIONAL FABRICATION Methods In THE ConteXt OF TEACHING PROTOTYPING FOR ENGINEERING DESIGN
}

\author{
Patrick Dumond \\ University of Ottawa \\ pdumond@uottawa.ca
}

\begin{abstract}
The introduction of Makerspaces across Canada has made it much easier for universities to provide engineering students the opportunity to "close the loop" on engineering design, by giving students the means to implement their projects. However, with the introduction of newer rapid prototyping technologies such as $3 D$ printing, students forget or are unware that traditional fabrication technologies can often be more efficient then these new technologies, depending on the situation.

This paper discusses the development and sustainability of traditional fabrication methods through the development of low cost dedicated facilities used in interdisciplinary engineering design courses. The introduction of these traditional fabrication methods have proven to increase the efficiency, creativity and critical thinking of engineering students related to the development of quick and iterative prototypes for their engineering designs. However, much work remains to be done if these facilities are to be optimized and sustained in this new engineering education paradigm.
\end{abstract}

Keywords: Engineering design; prototyping; traditional fabrication methods; makerspace; project based learning.

\section{INTRODUCTION}

Learning to implement designs is an important step in any engineer's education. Unfortunately, depending on the discipline, this step can be very resource intensive and has been slow to find widespread inclusion in engineering design courses across the country [1]. However, as project-based learning has increased in popularity, many universities have opted to introduce practical laboratory sessions on prototyping in order to complete the design cycle [2]-[4]. While some programs still rely exclusively on basic materials such as cardboard and toy construction kits, makerspaces have begun showing up on many campuses or in partnership with local communities [5], [6]. These makerspaces have made use of relatively new and innovative rapid prototyping technologies which have seen a significant reduction in cost over the past few years. These include 3D printers, laser cutters, CNC routers and other computer-controlled fabrication equipment.

While word of mouth has increased the number of students who have sought to actively improve their practical technical skills, this has not been enough to mobilize the entire student body. Therefore, the Faculty of Engineering at the University of Ottawa has sought to include the teaching of practical prototyping skills as part of its new first and second year interdisciplinary engineering design courses [7]. With access to the newly created Makerlab (adjacent to the openly accessible Makerspace), many students have come to understand the added difficulty inherent in the implementation of their designs.

Due to the success of these new courses and an increased emphasis on hands-on learning through various initiatives, the thriving maker culture in our engineering program has grown tremendously. This maker culture and accessibility to these rapid prototyping technologies have had a significant impact on student interest and engagement in other aspects of our engineering programs as well. This has been demonstrated by a significant increase in the number of students involved in extracurricular activities such as engineering competitions, community outreach and active participation in many other faculty initiatives [8].

These new rapid prototyping technologies have proven themselves to be a boon to the revitalization and update of our faculty and programs, demonstrating many positive attributes and benefits worthy of the additional allocation of resources. Nonetheless, as excitement continues to grow, a new problem has surfaced with respect to engineering design and prototyping directly related to these new prototyping technologies. The problem can be summarized in a statement which is often repeated to undergraduate students taking these new interdisciplinary design courses and can be summarized as follows: if you are going to make a flat plastic plate with a hole in it, buy a sheet of plastic, cut it to size and drill a hole in it. Don't 
$3 D$ print it! To which the students inherently respond: oh yeah, that would have saved me a lot of time! As with any new and fancy technology, excitement can quickly dissipate knowledge of what methods and technologies already exist for solving many problems efficiently.

In an attempt to rectify this problem, this paper will discuss the introduction of traditional fabrication techniques such as drill presses, band saws, mills, lathes and sheet metal work in the context of teaching prototyping for the purposes of completing the design cycle in undergraduate engineering design courses.

\section{INFRASTRUCTURE DEVELOPMENT}

\subsection{Background}

Over the last 150 years, engineering education has changed significantly. In most cases, early engineers would apprentice in the field, spending most of their time doing "hands-on" training. Later on, as the need for engineers increased, universities began offering engineering programs. These early programs involved mostly "shop" time, where students would spend their time designing and building products or learning to use engineering tools necessary to complete field work. The fabrication of these products often required knowledge of the use of traditional machine shop equipment. Therefore, learning to use these tools was a vital part of engineering programs at the time. As European educators, such as Stepan Timoshenko and Theodore von Kármán came to North-America, engineering analysis became a staple of engineering education, as more and more time was spent in the classroom learning theory based on mathematics and science. As the number of engineering students increased over the years, so did the number of hours spent in the classroom rather than the shop. The reason for this is in part due to the increasing amount of engineering science that was being taught in the programs and in part due to the difficulty in managing shop resources for a larger number of students. In fact, classroom teaching made it significantly easier to manage large engineering cohorts. After world war two, an emphasis was placed on researching fundamental science, as well as its application, further enhancing classroom teaching for engineers. However, as the new millennium approached, frustration grew regarding the lack of "hands-on" skills of graduating engineering students. Moreover, a shift towards greater concern for satisfying the needs of industry enhanced this frustration. This fueled significant changes regarding laboratory and design skills being taught at Canadian universities. Of course, these large institutions have been slow to adapt to this new paradigm shift.

In most cases, universities across Canada have been slowly revising their engineering programs to include more engineering design. Many have waited for the push from Engineers Canada and associated Canadian
Engineering Accreditation Board to take serious action [9]. Recently, the University of Ottawa has made a tremendous effort to revamp engineering design throughout its engineering programs by creating common first and second year interdisciplinary project based design courses [7]. These are followed up with program specific engineering design courses in third and fourth year [10], as well as optional interdisciplinary engineering design courses available to senior students [8]. In many cases, these new course rely heavily on project based learning, which is quickly gaining traction as an important tool in engineering education [11].

\subsection{Completing the Design Cycle}

To ensure that students understand the full implications of engineering design when using project based learning, it is important that students "complete the design cycle". For engineering design, "completing the design cycle" implies that their designs must be implemented. In most cases, this means that prototypes must be built. Of course, the implementation of designs can often be cost prohibitive due to the high cost per student of materials and equipment often necessary for the fabrication of prototypes.

With the recent innovations and price decrease of rapid prototyping equipment such as $3 \mathrm{D}$ printers and laser cutters, prototyping has once again become financially reasonable. Many institutions now include prototyping by using these technologies. However, through their excitement, students easily forget that many traditional fabrication techniques remain the most efficient way to create their prototypes. A need clearly exists to provide students with a broader understanding of technologies available for creating physical prototypes.

\subsection{Makerspace}

Access to a traditional machine shop (metal, plastic and wood fabrication) has always existed in the Faculty of Engineering at the University of Ottawa. These facilities have generally been staffed with technical personal and have been used for research. Over the years, a limited number of students have been trained to use machines such as mills and lathes and have been allowed to work on projects under strict supervision. In 2014, the Faculty developed its first Makerspace, which included mostly newer and significantly cheaper rapid prototyping technology [6], as can be seen in Fig. 1.

From the outset, this makerspace was developed to be freely accessible by all students and the surrounding community at no cost. The space was and continues to be fully funded by external grants and donations. Moreover, the space is fully run by students, which includes the training of new staff, the day to day operations, as well as the delivery of all workshops. Every year, a new student is chosen to manage the space from a group of students who have significantly participated in the day to day 
activities of the space in the previous year. Although this student run model continues to be tweaked, it has thus far proven to work very well, providing students invaluable experience which would otherwise not be possible through a typical engineering program.

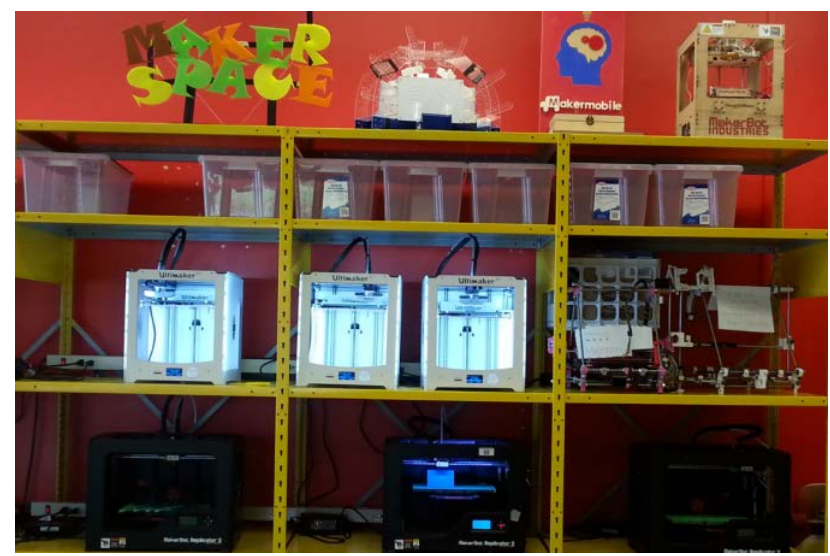

Fig. 1. Makerspace rapid prototyping equipment

To accommodate the higher and stricter demands required of the new first and second year design courses, a new space has been developed, known as the Makerlab, which is exclusively used to train students involved in these courses and provide them with the basic resources necessary for working on their prototypes.

With the advent of the Makerspace and Makerlab in the Faculty of Engineering at the University of Ottawa, as well as the use of these spaces as an official part of the new design courses, a widespread and significant increase in student participation was observed throughout all engineering programs. However, after just a single term of these new project based and hands-on design courses, it became immediately clear that students were limiting their creativity to the new rapid prototyping technologies being made available through these spaces. In fact, almost all projects relied on 3D printed components, laser cut MDF and Arduino microcontrollers. In many cases, students spent hours, for example, 3D printing rectangular boxes as enclosures for their Arduino's only to see their prints fail before completion. Not only did this tax the resources in the Makerspace/Makerlab, but it did not help students understand the rapid and iterative nature of modern prototyping, due the severely inefficient tools being used.

\subsection{Machine Shop}

To address these concerns, a new traditional machine shop training facility, known as the Manufacturing Training Centre (MTC), was developed, as can be seen in Fig. 2. This new facility aimed to train students on the use of traditional metal and plastic fabrication machines such as mills, lathes, bandsaws, drill presses, as well as a number of tools related to sheet metal work. In doing so, it was hoped that students would get a broader understanding of the new and old technologies that exist for the fabrication of physical prototypes and also making these technologies accessible to the students. In this case, MTC machine training also became an official part of the new design courses.

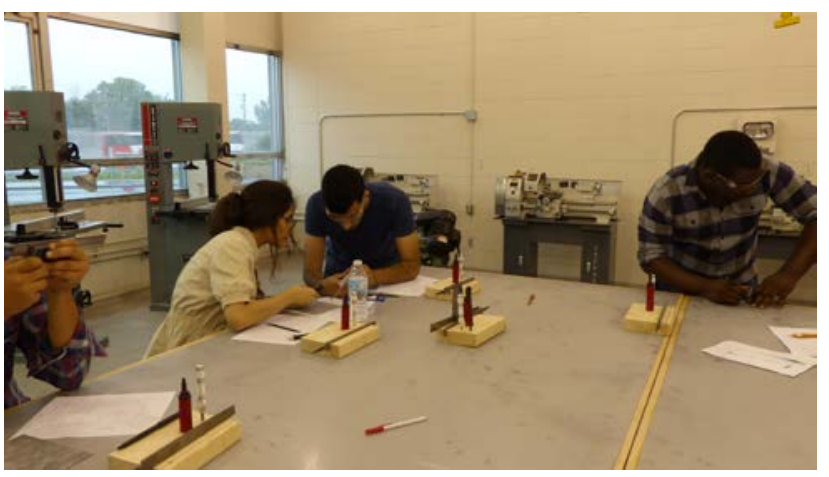

Fig. 2. Manufacturing Training Centre

To keep costs down and to provide a large number of machines to students, smaller machines were chosen, with most able to run on $120 \mathrm{~V}, 15 \mathrm{~A}$ circuits. This also minimized the cost associated with the electrical circuit infrastructure required. These machines proved to be adequate for the introductory nature of most training programs. The initial MTC setup included the following equipment:

- $4 \times$ Craftex lathe (CX706)

- $6 \times$ Craftex mill (CX601 and CX 611)

- $3 \times$ King drill press (KC-118FC)

- $2 \times$ King vertical bandsaw (KC-1700WM-VS)

- $1 \times$ King horizontal bandsaw (KC-712BC)

Furthermore, a sheet metal brake, metal shear, metal hand shear, corner notcher and spot welder were also repurposed for this space. Finally, miscellaneous metal working and sheet metal working hand tools (e.g., machine squares, scribes, deburring tools, files, etc.) and machine tooling were purchased to complete the training essentials.

To supplement this new space, the Brunsfield Centre (Fig. 3), a student project space, was redeveloped to include many new industrial grade fabrication machines. As with the Makerspace, the Brunsfield Centre was made to be freely accessible by all students at no cost. Therefore, with properly recorded training in the MTC, students can use the equipment in the Brunsfield Centre to work on any project.

\section{FABRICATION SPACES}

\subsection{Manufacturing Training Centre}

At the outset, the MTC was to be used as part of both the first and second year interdisciplinary design courses. In the first year course, students learn the basics of metal work by doing layouts and working with sheet metal. The 
drill press and the bandsaws are also introduced. In the second year course, students are introduced to the mill and lathe through the creation of a robot. In total, 700 students were trained to operate the equipment and tools safely in the first two years of this new offering, a number which is expected to double for the 2018-2019 school year. For those who are interested in improving their skills, advanced workshops are available throughout the year to be completed on the students' own time.

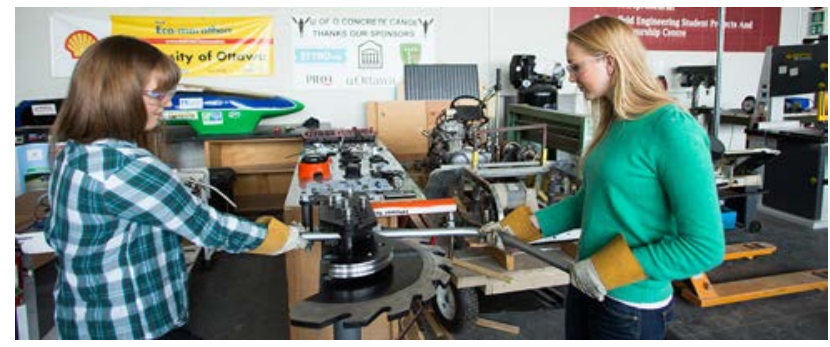

Fig. 3. Brunsfield Centre

The space is organized and managed by students with the help of a technical staff. Training workshops so far have been given by both students and the technical staff member. Training the trainers has occurred organically, through experienced students. The presence of a technical staff in the space has proven to be vital in the early setup of the space. This is especially true of machine maintenance, which students tend to forget. However, as time passes and formal processes are developed, students have taken on more responsibility in the day to day operations of the MTC.

\subsection{Brunsfield Centre}

On the other hand, the Brunsfield Centre was developed to be openly accessible to students to work on their projects at times convenient to them. The Brunsfield Centre provides students with a comprehensive set of equipment, including full-size fabrication machinery, power tools and hand tools. Since the risk of accidents or injury are significantly higher than with the equipment provided in the Makerspace, mandatory training on all the equipment is necessary in order to use it and experienced student shop supervisors are present at all times while the equipment is being used.

As with the Makespace, the Brunsfield Centre is fully managed, organized and run by the students, providing them with invaluable experience not possible through the regular engineering program

\subsection{Safety Protocols}

Since the safety of students is paramount, the development of stringent safety protocols has been necessary to ensure the proper functioning of the space. Supervisors are chosen from students who have not only demonstrated their proficiency in using the equipment, but who have also demonstrated high levels of responsibility. The supervisors must then follow training designed specifically to teach the students what to look out for in the use of the equipment by others. Moreover, they must follow lab safety training and complete all government mandated training. Furthermore, supervisors must complete daily risk assessments to ensure the spaces are safe to work in. If issues are discovered, they must rectify the situation or make a plan which will ensure the spaces are used safely or returned to a safe state.

Since the risk of untrained students using the equipment is potentially high, a new electronic sign in system was created, allowing students to tap their student card upon entry. This sign-in system is linked to an online database which gives shop supervisors an instantaneous overview of trainings completed by the student, as well as hours logged on specific equipment. This information is vital to ensure that students using the space are qualified to do so and do so safely with varying levels of supervision.

Of course, ill-maintained equipment poses a safety risk to the user and also to those within the immediate environment. For this reason, equipment maintenance schedules and protocols have been developed and are continuously monitored for improvement to ensure that all risks regarding malfunctioning equipment is minimized. While at any given time throughout the year a student is specifically tasked with ensuring the equipment is maintained on a weekly, monthly and quarterly basis, supervisors are also responsible for verifying the working condition of equipment on a daily basis.

\subsection{Sustainability}

Overall, the University of Ottawa has managed to develop the MTC and Brunsfield Centre for approximately $\$ 150 \mathrm{k}$ (not including equipment which was already available on campus at the beginning of the project). However, sustaining these spaces has proven to be more difficult than what was found to be required for the Makerspace/Makerlab. The ongoing maintenance and tooling cost of traditional fabrication equipment is higher than newer low cost rapid prototyping technologies and more supervisors and trainers are required for the proper functioning of these new prototyping spaces due to the extra safety concerns related to this equipment.

To offset these costs, the Brunsfield Centre has begun selling raw material, such as metal, wood and plastic, as well as hardware, at a small profit. This not only helps sustain the spaces, but is also appreciated by many students who do not have the means to pickup material and hardware on their own. However, much more work remains to be done if these spaces are to survive for many years to come. 


\section{DISCUSSION AND CONCLUSION}

\subsection{Discussion}

Through the availability of these spaces for the project based engineering design courses, students have significantly improved the variety and functional quality of their prototypes. Moreover, the number of prototyping iterations for many groups seems to have gone up as well. In many cases, excitement regarding the students' prototypes has increased and many students have continued to improve their prototype fabrication skills even after the end of the course or have moved on to other interesting projects which have also been implemented. This sets up the students well for becoming lifelong learners. As engineering educators, the availability of these spaces has also made it much easier to discuss and explore prototyping options with students regarding their engineering designs. While these new traditional fabrication facilities have certainly brought a significant amount of positive energy to the Faculty of Engineering's programs, much work remains to be completed if the use of these spaces is to be optimized and if these spaces are to be sustainable.

Firstly, the current safe configuration for the use of these spaces is a maximum of 24 students at a time. Even at 700 students, this has proven to be difficult to manage from a scheduling perspective. If the number of students are to double, a significant increase in the capacity would be required. Luckily, through popularity of these very initiatives, the University of Ottawa has managed to secure funding for a brand new building which will house an entire floor for these engineering design spaces. This new building, which will be fully operational for the fall of 2018, will provide the drastically needed increase in capacity.

Additionally, the continuous supply of supervisors and trainers required to operate these spaces has proven to be difficult early on. However, as time has gone by and with the increased interest of students for "hands-on" activities, a progressively larger number of students have shown interest in ensuring they gain the required experience. Nonetheless, to be sustainable, a formal training process should be developed, beginning with first year students, to ensure that a continuous supply of senior students are available to take on these critical (and paid) roles.

The training projects should also be continuously revised and updated to ensure that students are learning as effectively and as safely as possible.

Other problems also needed to be overcome in the development of these spaces early on. These problems included the development of training and maintenance programs. A lack of traditional fabrication experience in the student population made it somewhat difficult at the beginning. Luckily, a technical staff was made available to aid in the early development of these spaces. Since one of the primary goals of these initiatives is to let students manage and operate these spaces themselves, the implication of this technical staff member will likely eventually be reduced. However, much work remains to be done to ensure that appropriate processes and protocols are put in place to ensure that the spaces can continue to be sustained via the student population.

Another unexpected problem that was faced early on involved the direction of early fabrication training. Since experienced students were used in the development of early training programs, even introductory training placed emphasis on trying to make machinist out of every student in the Engineering program. While this would be excellent experience for the students, it would not be feasible, even at 700 students, let alone more. Moreover, it was clear early on that not all student showed the same enthusiasm for machining. For these reasons introductory training emphasis was shifted towards ensuring that all students understood what technologies were available in the fabrication of prototypes, how each technology could be used for maximum efficiency, and most importantly, how the equipment could be used safely. Of course, advanced training was also developed for those who demonstrated a keen interest in machining.

The optimal use of these design spaces has also proven to be difficult, as heavy use is made of the facilities at the beginning of the term to train students so they can then use the spaces to fabricate their prototypes and at the end of the term when project deadlines are looming. Meanwhile, the spaces usually experience lulls during the midpoint of the term and during exams. Further work is required if the use of these spaces is to be optimized.

An unexpected outcome of these new spaces, was the boon it provided to the engineering competition teams, as can be seen in Fig. 4. Not only did it provide these students with significantly better resources for completing their projects, but it also provided the teams with an increased supply of useful students capable of actively participating in the projects from day one.

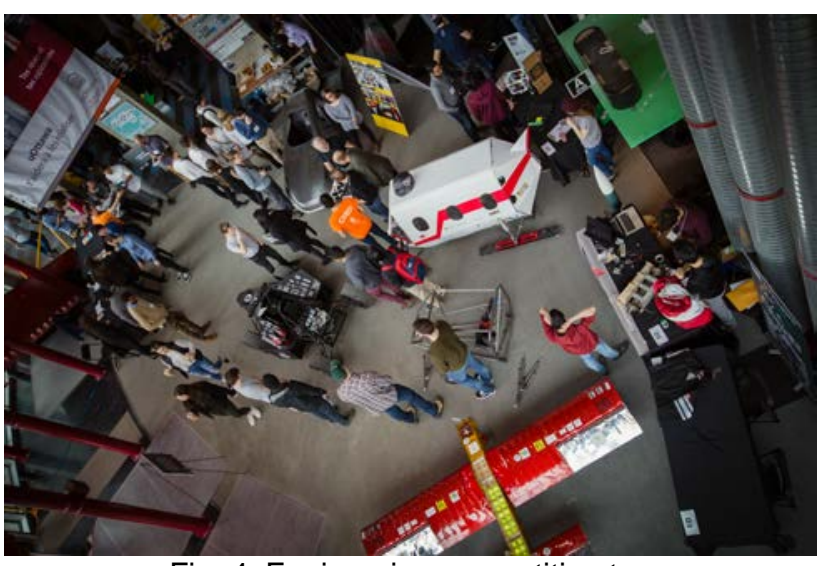

Fig. 4. Engineering competition teams 


\subsection{Conclusion}

The development of traditional prototype fabrication facilities has proven to be highly beneficial in the education of students who have come to know newer rapid prototyping technologies as the go to for prototype fabrication. In fact, it has provided students with a broader range of tools which has allowed them to be more efficient, creative and critical in their engineering designs. However, setting up and sustaining traditional fabrication spaces and teaching these techniques has proven more difficult than maintaining a rapid prototyping makerspace for many reasons. These include: cost of equipment and materials, availability of trainers, equipment maintenance, and most importantly, safety. Each one of these difficulties has been addressed with a heavy emphasis on student involvement and lifelong learning. Moreover, a fine balance must be considered between training engineers who understand the broad range of technologies available for prototyping and highly skilled machinist. Many valuable lessons in the development of this program were learned along the way and this initiative is beginning to bear fruit in creating engineers better prepared to prototype their designs efficiently and creatively.

\section{References}

[1] C. L. Dym, A. M. Agogino, O. Eris, D. D. Frey, and L. J. Leifer, "Engineering Design Thinking, Teaching, and Learning," Journal of Engineering Education, vol. 94, no. 1, pp. 103-120, Jan. 2005.

[2] R. Carrick, A. Czekanski, and M. R. Ha, "Building a More Complete Design Experience: Philosophies and Reflections from a Second Year Mechanical Engineering Design Project Course," Proceedings of the Canadian Engineering Education Association, Jun. 2016.
[3] W. Stiver, "First Year Engineering Design Guelph's Teddy Bear Wheel Chair Experience,” Proceedings of the Canadian Engineering Education Association, Aug. 2015.

[4] V. Komisar, A. Flood, N. Walji, J. Foster, and R. Irish, "Teaching Credible Validation and Verification Methods to a Large, Multidisciplinary First-year Engineering Design Class,” 1, 2017.

[5] M. Wlodyka and B. Tomberli, "Incorporating a Community-based Rapid Manufacturing Facility into a First Year Engineering Design Course,” 1, 2016.

[6] M. Galaleldin, F. Bouchard, H. Anis, and C. Lague, "The Impact of Makerspaces on Engineering Education," Proceedings of the Canadian Engineering Education Association, 2016.

[7] M. Galaleldin, H. Anis, P. Dumond, and D. Knox, "Scaffolding Strategies for Teaching Engineering Design in a Collaborative Project-based Learning Environment," 1, 2017.

[8] Patrick Dumond, "A Course Based Approach to Recognizing Student Efforts in Engineering Design Competitions," 1, 2017.

[9] CEAB, "2016 Accreditation Criteria and Procedures,” Engineers Canada, 2016.

[10] P. Dumond and E. Lanteigne, "From Mechanical Engineering Capstone Design to Design Implementation," Proceedings of the Canadian Engineering Education Association, Jun. 2016.

[11] M. Frank, I. Lavy, and D. Elata, "Implementing the Project-Based Learning Approach in an Academic Engineering Course," International Journal of Technology and Design Education, vol. 13, no. 3, pp. 273-288, Oct. 2003. 